\title{
Optimization of Energy Efficiency in Massive MIMO System Review Using Wolfe Method
}

\author{
Preeti Sharma ${ }^{1, *}$ and Dr. D.C. Dhubkarya ${ }^{2}$ \\ 1Faculty of Engineering and Technology, Agra College, Agra, India; preetifet@gmail.com \\ 2Bundelkhand Institute of Technology and Science, Jhansi, India; dcd3580@yahoo.com
}

\begin{abstract}
Objectives: This research study presents $5 \mathrm{G}$ technology of Massive MIMO system for its spectral and energy efficiency benefits for single antenna equipped receiver for maximal ratio combiner and zero-forcing precoding techniques in perfect and imperfect CSI conditions. We consider a cellular system for single and multiple cells. We show optimization for energy efficiency at constant spectral efficiency. Methods/statistical analysis: Massive MIMO system implementation with M number of the antenna at the base station and singe antenna at the receiver. Study of receiver's precoding techniques like maximal ratio combiner and zero-forcing for energy and spectral efficiency. Wolfe optimization technique of nonlinear programming used to maximize energy at constant spectral efficiency. Findings: The resulting graph shows that massive MIMO improves spectral and energy efficiency for both MRC and ZF precoding. Energy Efficiency performance for ZF and MRC precoding techniques with the number of antennas in uplink communication of massive MIMO system gives that user power requirement reduces as a number of antennas grows. In perfect CSI condition, it reduces by 1/M and imperfect CSI condition scale down by factor $1 / \sqrt{ } \mathrm{M}$. Matlab contour graph gives optimized values for energy efficiency for constant spectral efficiency. Application/improvements: efficient design of cellular communication for spectral and energy usage. Soft computing techniques can give batter results.
\end{abstract}

Keywords: Massive MIMO, Energy Efficiency, Optimization, Wolfe

\section{Introduction}

Cellular communication is the fastest growing network which is making our world easier providing any time anywhere services. Current internet system using $4 \mathrm{G}$ and advanced LTE with OFDM-MIMO system providing high data rate and reliable connection. Our multimedia applications like CCTV surveillance, video conferencing and stored video play needs high quality of service and power consumption.

Researcher moving towards next-generation wireless systems as massive MIMO, millimeter-wave,,$\underline{1,2}$ NOMA. Massive MIMO system referred to as large numbers of antennas arrangement MIMO, is a form of multiuser MIMO in which the number of antennas present at the base station is much larger than the number of user equipment per signaling resource. Here we are discussing massive MIMO regarding its throughput energy efficiency and spectral efficiency. $1, \underline{3}$

Massive MIMO System has a very large number of antenna array at the base station (approximately 50 to 200) These large number of antennas at the base station gives huge spatial multiplexing gain. It increases cellular network capacity by several folds to the current system (LTE, 4G). Massive MIMO contains an array of physically small nondirective cheap antennas at the base station which serves several user terminals are with a single antenna. In this number of users are less in comparison to the number of antennas.,$\underline{5}$

In this communication system, a huge number of links are present between BS to the user, so it decreases interference and fading effect. This study shows perfect and imperfect CSI for ZF and MRC precoding performance in terms of spectral and energy of the

${ }^{*}$ Author for correspondence 
massive MIMO system. We also find the optimized value of energy efficiency for a given set of variables.., 7

In the massive MIMO cellular system, researchers are proposing their solution to increase the data rate, coverage area and energy efficiency. Power generation from conventional resources are limited and from the nonrenewable resource are much costlier. There are three ways to increase energy efficiency. $\underline{8}$

1. Decrease energy consumption and decrease data rate

2. Decrease energy consumption and increase the data rate

3. Increase energy consumption and increase the data rate

Here, we are discussing some conclusions which are drawn by researchers.

Ngo et al consider the uplink MU-MIMO system in perfect CSI and Imperfect CSI condition with linear precoding for calculating spectral and energy efficiency. Both efficiency increase as we increase the number of antennas at the base station. In perfect CSI power-law scales down by $1 / \mathrm{M}$ and in imperfect CSI it scales down by $1 / \sqrt{M}$ . Spectral efficiency depends on the number of antennas as well on the required power. It shows the number of points on the graph between spectral and energy efficiency for a constant number of antenna and user tradeoff.

D. Ha et al shows the relation between energy and spectral efficiency, including circuit power consumption. The power consumption model includes circuit power and transmitting power according to the requirement of signal to noise ratio in different channel conditions.

Z. Liu et al gave a solution to select required sum-capacity and energy consumption by selecting a number of the antenna at the base station using mixed-integer continue variable multi-objective optimization using weighed sum particle swarm optimization and normal boundary intersection particle swarm optimization algorithm on zero-forcing and maximal ratio combining precoding.

E. Brijson et al proposed a massive MIMO system using small cell gives batter coverage as well as energy efficiency. The graph shows comparative energy efficiency response with spectral efficiency by base station antenna density.

\section{System Model}

\subsection{Massive MIMO Model}

When base station consists of $\mathrm{M}$ number of transmitting antennas $(\mathrm{M} \gg K)$, it increases spatial multiplexing and reduces noise effect and small-scale fading. The researcher shows that tens of transmit antennas give higher data throughput from a conventional MIMO system. Massive MIMO has three key aspects that firstly a number of antennas at the base station are much higher than the user terminal with a single antenna in a single cell. $4, \underline{5}$ Second the base station assesses the uplink channel on the grounds that the required pilot carrier is relative to a number of users in a single cell. The third one simple linear precoding is employed for multiplexing on the uplink or downlink. $-\underline{-11}$

The Multi-user Massive MIMO system is shown in Figure 1.

Mathematical Modeling of Massive MIMO system:

$$
\begin{aligned}
& M>>K>1, \\
& \bar{Y}=\sqrt{ } P_{u} G \bar{x}+\bar{n}
\end{aligned}
$$

$$
\begin{aligned}
& \bar{Y}=\left[\begin{array}{c}
y 1 \\
y 2 \\
-
\end{array}\right] \mathrm{G}=\left[\begin{array}{llll}
g 1 & g 2 & g 3 \ldots g k
\end{array}\right] \quad \bar{X}=\left[\begin{array}{c}
x 1 \\
x 2 \\
-
\end{array}\right] \\
& n=\left[\begin{array}{c}
n 1 \\
n 2 \\
-
\end{array}\right] G=H D^{\frac{1}{2}} \text { where }[\mathrm{D}]_{\mathrm{KK}}=\beta_{k} \\
& \frac{G G^{H}}{M}=H D^{\frac{1}{2}}\left[H D^{\frac{1}{2}}\right]^{H} \approx D \quad M>>K
\end{aligned}
$$

\subsubsection{Single Cell Massive MIMO System}

\subsubsection{Perfect CSI}

In single-cell consideration of M-MIMO system BS have the large number of antennas arranged with small spacing on a board. These antennas are small in size, formed in an array with inexpensive hardware. This arrangement reduced small scale fading and random

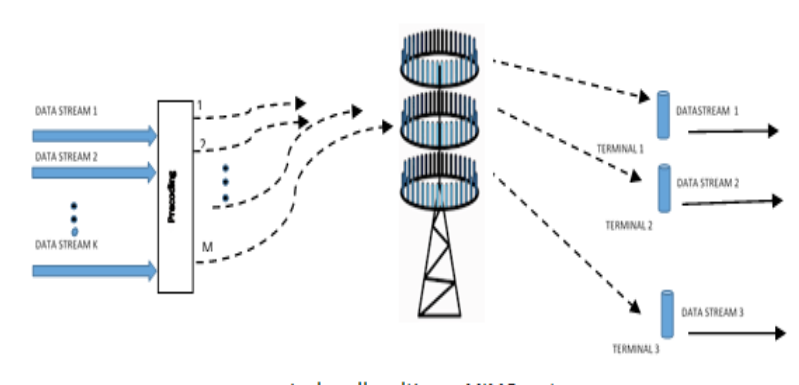

single cell multi user MIMO system

Figure 1. Multi-user Massive MIMO system. 
channel vectors between user and base station become pair wisely orthogonal. The advantage of maximum ratio combining (MRC) is compared with ZF is not only its calculus clarity--- the received signals multiply with the associated channel response. But also it can be performed in a disturbed manner at each individual antenna unit. While ZF also works sufficiently well for a standard and medium numbers antenna system, MRC generally for low regime. $\underline{5}$ Received signal at matched filter using a linear decoder

$$
\begin{aligned}
r_{m r c} & =G^{H} y \\
r_{Z F} & =\left(G\left(G^{H} G\right)^{-1}\right)^{H} y \\
r_{M M S E} & =\left(G\left(G^{H} G+\frac{1}{p_{u}} I_{k}\right)^{-1}\right)^{H} y
\end{aligned}
$$

$M \times 1$ received vector $y_{k}$ at $B S$ from the kth user as

$$
Y_{K}=\sqrt{p_{u} g_{k} x_{k}}+\sqrt{p_{u}} \sum_{i=1 i * k}^{K} g_{i} x_{i}+n
$$

The matched filter receiver for the kth user is given as

$$
r_{m r c}=\frac{g_{k}{ }^{H}}{\left\|g_{k}\right\|}\left(\sqrt{p_{u}} g_{k} x_{k}+\sqrt{p_{u}} \sum_{i=1 i \neq k}^{K} g_{i} x_{i}+n\right)
$$

Now signal to noise interference ratio given by

$$
\begin{gathered}
\operatorname{SINR}=\frac{p_{u}\left\|g_{k}\right\|^{2}}{1+p_{u} \sum_{i=1}^{K}{ }_{i \neq k}\left|\frac{g_{k}{ }^{H}}{\left\|g_{k}\right\|} g_{i}\right|^{2}} \\
\frac{g_{k}{ }^{H}}{\left\|g_{k}\right\|} n \sim N(0,1), \frac{g_{k}{ }^{H}}{\left\|g_{k}\right\|} g_{i} \sim N\left(0, \beta_{i}\right)
\end{gathered}
$$

By putting values

$$
\begin{gathered}
\operatorname{SINR} \rightarrow \frac{p_{u}\left\|g_{k}\right\|^{2}}{p_{u} \sum_{i=1 \text { i }}^{K} \beta_{i}+1} \text { Each user power } p_{u}=\frac{E_{u}}{M} \\
\operatorname{SINR}=\frac{E_{u} \frac{\left\|g_{k}\right\|^{2}}{M}}{\frac{E_{u}}{M}\left(\sum_{i=1, i \neq k}^{K} \beta_{i}\right)+1}\left\|g_{k}\right\|=M \beta_{K}
\end{gathered}
$$

By channel capacity Theorem

For lower bound

$\mathrm{R} \geq \mathrm{C}=\mathrm{Blog}_{2}(1+\mathrm{SINR})$

$$
R_{k}^{m r c}=\log _{2}\left(1+\frac{\frac{E_{u}}{M} \beta_{K}(M-1)}{\frac{E_{u}}{M}\left(\sum_{i=1, i \neq k}^{K} \beta_{i}\right)+1}\right)
$$

If the number of antenna $\mathrm{M} \rightarrow \infty$

SINR $\rightarrow E_{\mathrm{u}} \beta_{\mathrm{K}}$

because $\frac{E_{u}}{M}\left(\sum_{i=1, i \neq k}^{K} \beta_{i}\right) \rightarrow 0$

Zero Forcing Receiver:

We can calculate the rate for $\mathrm{ZF}$ linear decoder

$$
\begin{gathered}
r_{Z F}=\left(G\left(G^{H} G\right)^{-1}\right)^{H}\left(\sqrt{p_{u}} g_{k} x_{k}+\sqrt{p_{u}} \sum_{i=1 i \neq k}^{K} g_{i} x_{i}+n\right) \\
R_{k}^{Z F}=\log _{2}\left(1+\frac{E_{u}}{M}(M-K) \beta_{K}\right)
\end{gathered}
$$

If $\mathrm{M} \rightarrow \infty$

The lower bound rate will tend to

$$
R_{k}^{Z F}=\log _{2}\left(1+E_{u} \beta_{K}\right)
$$

And upper bound will be

$$
R_{k}^{Z F}=\sum_{i=1}^{K} \log _{2}\left(1+E_{u} \beta_{K}\right)
$$

\section{2) Zero}

\subsubsection{Imperfect Channel State Information}

In imperfect state matrix $\mathrm{G}$, had been estimated at the BS Pilot symbols are transmitted are Table 1 . Let assume $\mathrm{T}$ is the length (Time $\times$ Bandwidth) of the coherence interval and $\tau$ is the number of symbols used for orthogonal pilot sequence used by $\mathrm{K}$ users may be shown as $\tau$ by $\mathrm{K}$ matrix $\sqrt{P_{n}} \varnothing(\tau \geq K)$

$$
\begin{gathered}
\varnothing^{H} \varnothing=I_{K} \\
P_{n} \triangleq \tau P_{u}
\end{gathered}
$$

From equations, the achievable rate in Imperfect CSI for $\mathrm{ZF}$ and MRC can be given as below.

$$
R_{I m p, K}^{M r c}=\log _{2}\left(1+\frac{P_{u} \tau(M-K) \beta_{K}{ }^{2}}{\left(\tau P_{u} \beta_{K}+1\right) \sum_{i=1, i \neq K}^{K} \beta_{i}+(\tau+1) \beta_{K}+\frac{1}{P_{u}}}\right)
$$


Table 1. Symbol notation used in paper

\begin{tabular}{|c|c|}
\hline Symbol & Parameter \\
\hline M & Base station antennas in a single cell \\
\hline $\mathrm{K}$ & User terminal with a single RF chain in a cell \\
\hline $\mathrm{Y}$ & received vector at $B S$ of $M \times 1$ \\
\hline $\mathrm{P}_{\mathrm{u}}$ & User transmitted power (average) \\
\hline G & $\begin{array}{l}\text { Channel matrix of } \mathrm{M} \times K \text { between the base } \\
\text { station and users }\end{array}$ \\
\hline$\sqrt{P_{u}} X$ & $\begin{array}{l}\text { Vector of symbols simultaneously transmitted to } \\
\text { the K users }\end{array}$ \\
\hline $\mathrm{N}$ & $\begin{array}{l}\text { Gaussian noise vector of additive white noise with } \\
\text { zero mean }\end{array}$ \\
\hline $\begin{array}{l}\mathrm{g}_{\mathrm{mk}} \triangleq \\
{[\mathrm{G}]_{\mathrm{mk}}}\end{array}$ & $\begin{array}{l}\text { Channel coefficient between } \mathrm{m}^{\text {th }} \text { antenna and } \mathrm{k}^{\text {th }} \\
\text { user }\end{array}$ \\
\hline $\mathrm{h}_{\mathrm{mk}}$ & $\begin{array}{l}\text { Fast fading coefficient from the } \mathrm{k}^{\text {th }} \text { user to the } \mathrm{m}^{\text {th }} \\
\text { antenna of the BS }\end{array}$ \\
\hline$\sqrt{\beta_{K}}$ & $\begin{array}{l}\text { Geometric attenuation and shadow fading } \\
\text { constant }\end{array}$ \\
\hline $\mathrm{R}$ & Received vector after using the linear decoder \\
\hline $\mathrm{H}$ & $\begin{array}{l}\text { Small scale fading matrix of } \mathrm{M} \times \mathrm{K} . \mathrm{H} \text { are i.i.d. } \\
\text { random variables with } 0 \text { mean and } 1 \text { variance. }\end{array}$ \\
\hline $\mathrm{D}$ & Diagonal matrix of $\mathrm{K}$ by $\mathrm{K}$ \\
\hline $\mathrm{E}_{\mathrm{u}}$ & Total user power \\
\hline $\mathrm{L}$ & Number of cell in multi-cell configuration \\
\hline$\eta_{p}^{v}$ & Energy efficiency in bits per joule \\
\hline$R_{k}^{v}$ & $\begin{array}{l}\text { Channel rate of the kth user using } \mathrm{v} \text { type of linear } \\
\text { precoding method }\end{array}$ \\
\hline
\end{tabular}

$R_{I m p, K}^{Z F}=\log _{2}\left(1+\frac{P_{u} \tau(M-1) \beta_{K}^{2}}{\left(\tau P_{u} \beta_{K}+1\right) \sum_{i=1, i \neq K}^{K} \frac{P_{u} \beta_{i}}{\tau P_{u} \beta_{i}+1}+\tau P_{u} \beta_{K}+1}\right)$

In both cases, imperfect power-law reduce each user power in terms $1 / \sqrt{M}$ of and if we take

$\mathrm{M} \rightarrow \infty$ both equation will give $R_{\operatorname{Imp}, K}^{V} \rightarrow \log \left(1+\tau{\beta_{K}}^{2} P_{u}^{2}\right)$

\subsubsection{Multiple Cell M-MIMO System}

The Multiple cells equipped with a massive MIMO system are shown in Figure 2.

The uplink analysis in multiple cells as L cells of massive MIMO system sharing the same frequency band. In each cell BS equipped with a very large number of antennas and $\mathrm{K}$ users with a single antenna. When BS has perfect

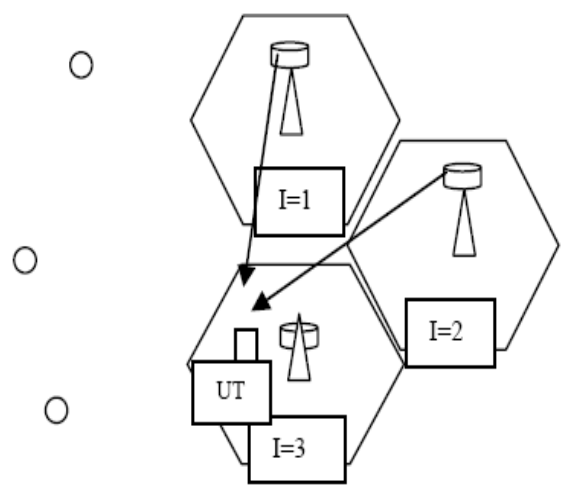

Figure 2. Multiple cells equipped with a massive MIMO system.

CSI that means it knows $\mathrm{G}$. Let $\mathrm{M}$ by $\mathrm{K}$ linear detector matrix depends on the channel $\mathrm{G}_{\mathrm{l} \text {. }}$. The $\mathrm{M}$ by 1 received vector at $\mathrm{BS}$ given by

$$
Y_{l}=\sqrt{ } P_{u} \sum_{i=1}^{L} G_{l i} x_{i}+n_{1}
$$

$\mathrm{i}=1$ to $\mathrm{L}$ number of cells

This equation indicates received signal $y_{1}$ at $l^{\text {th }}$ base station when the user is located at the ith cell.

$$
G_{l i}=H_{l i} D_{l i}^{\frac{1}{2}}
$$

Maximal ratio combiner: - in MRC linear detector received signal is

$$
\begin{gathered}
r_{l 1}=G_{l i}^{H} y_{l} \\
r_{11}=G_{l i}^{H}\left(\sqrt{ } P_{u} \sum_{i=1}^{L} G_{l i} x_{i}+n_{l}\right)
\end{gathered}
$$

If perfect channel sensing indication (CSI) is there then user 1 will have without loss of generality in cell 1 .

$$
\begin{aligned}
Y_{1} & =\sqrt{ } P_{u} g_{111} x_{11}+\sqrt{ } P_{u} \sum_{i=2}^{K} g_{1 l i} x_{1 i} \\
& +\sqrt{ } P_{u} \sum_{i=2}^{L} \sum_{i=2}^{K} g_{1 j i} x_{1 i}+n_{1}
\end{aligned}
$$

Using the matched filter at receiver for user 1

$$
\begin{aligned}
& r_{11}=\sqrt{P_{u}} \frac{g_{111}^{H}}{\left\|g_{111}\right\|} g_{111} x_{11}+\sqrt{P_{u}} \sum_{i=2}^{K} \frac{g_{111}^{H}}{\left\|g_{111}\right\|} g_{11 i} x_{1 i} \\
& +\sqrt{P_{u}} \sum_{i=2}^{K} \sum_{j=2}^{L} \frac{g_{111}^{H}}{\left\|g_{1 j i}\right\|} g_{1 j i} x_{j i}+\frac{g_{111}^{H}}{g_{111}} n_{1}
\end{aligned}
$$

Now for power requirement by the user can be calculated by SINR 


$$
\begin{gathered}
\operatorname{SINR}=\frac{P_{u}|| g_{111} \|}{\sqrt{P_{u}} \sum_{i=2}^{K}\left|\frac{g_{111}^{H}}{\left\|g_{111}\right\|}\right| g_{111} x_{1 i}+\sqrt{P_{u}} \sum_{i=2}^{K} \sum_{j=2}^{L}\left|\frac{g_{111}^{H}}{\| g_{111} \mid}\right| g_{1 j i} x_{j i}+1} \\
\operatorname{SINR} \rightarrow \frac{P_{u}\left\|g_{111}\right\|^{2}}{P_{u} \sum_{i=2}^{K} \beta_{11 i}+P_{u} \sum_{i=2}^{K} \sum_{j=2}^{L} \beta_{1 j i}+1}
\end{gathered}
$$

Gaussian random variable properties implied on variables we will take or channel hardening.

$$
\begin{aligned}
& \frac{g_{111}^{H}}{\left\|g_{111}\right\|} n_{1} \sim \mathrm{N}(0,1), \frac{g_{111}^{H}}{\left\|g_{111}\right\|} g_{11 i} \sim N\left(0, \beta_{11 i}\right) \\
& \frac{g_{111}^{H}}{\left\|g_{111}\right\|} g_{1 j i} \sim N\left(0, \beta_{1 j i}\right) \\
& \mathrm{R} \rightarrow \log _{2}(1+\mathrm{SINR})
\end{aligned}
$$

If the number of the antenna is $\mathrm{M}$ at the Base station provide power $E_{u}$ then each user power scaling as $E_{u} / M$.

$$
\begin{aligned}
& \text { Put } P_{u}=\frac{\mathrm{E}_{\mathrm{u}}}{M} \text { in equation } \\
& \qquad \text { SNIR } \rightarrow \frac{\frac{\mathrm{E}_{\mathrm{u}}}{M}\left\|g_{111}\right\|^{2}}{\frac{\mathrm{E}_{\mathrm{u}}}{M} \sum_{i=2}^{K} \beta_{11 i}+\frac{\mathrm{E}_{\mathrm{u}}}{M} \sum_{i=2}^{K} \sum_{j=2}^{L} \beta_{1 j i}+1}
\end{aligned}
$$

Pair wise orthogonality gives

$$
\text { SNIR } \rightarrow \frac{\frac{\mathrm{E}_{\mathrm{u}}}{M} \beta_{111} M}{\frac{\mathrm{E}_{\mathrm{u}}}{M} \sum_{i=2}^{K} \beta_{11 i}+\frac{\mathrm{E}_{\mathrm{u}}}{M} \sum_{i=2}^{K} \sum_{j=2}^{L} \beta_{1 j i}+1}
$$

Put Limit as $\mathrm{M} \rightarrow \infty$ in SINR

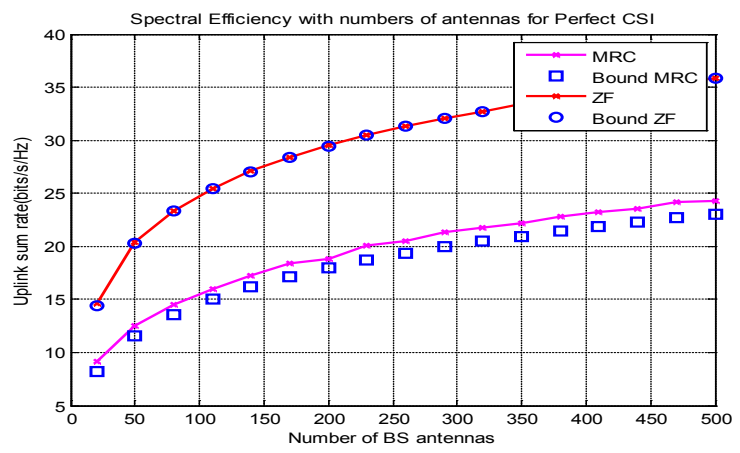

Figure 3. Spectral efficiency graph for perfect CSI with zero-forcing and maximal ratio combiner precoding technique.

$$
\begin{aligned}
& \mathrm{SINR} \rightarrow \beta_{111} \mathrm{E}_{\mathrm{u}} \\
& \mathrm{R} \rightarrow \log _{2}\left(1+\mathrm{E}_{\mathrm{u}} \beta_{1}\right)
\end{aligned}
$$

Thus the single-cell rate can be maintained by power scaling as $\mathrm{P}_{\mathrm{u}}=\frac{\mathrm{E}_{u}}{M}$ in the multicell system. In the massive MIMO system power of each user can decreases as the number of antennas $\mathrm{M}$ increases.

\subsection{Spectral Efficiency and Energy Efficiency Relation}

Energy efficiency $\left(\eta_{p}^{v}\right)$, defined the ratio of spectral use in bits/channel to power consumption in joule/channel. So energy efficiency will be in bits/joule. $\underline{12}-\underline{14}$

Energy efficiency[bits / joule]

$$
=\frac{\text { Data throughput }[\mathrm{bits} / \mathrm{s}]}{(\text { Energy consumption }[\text { joule } / \mathrm{s}])}
$$

Data throughput $=$ Spectral Efficiency $=R_{p K}^{V}=\sum_{k=1}^{K} R_{p k}^{V}$

$$
\text { Energy consumption }=P_{u}(\text { circuit power ignored })
$$

We assumed that transmitted data are modulated by OFDM. In perfect CSI uplink pilots are equal to the number of user sequence length chosen. We use $\mathrm{P}_{\mathrm{u}}=10$ $\mathrm{dB}$ and $\mathrm{K}=10$ users in perfect CSI condition and relation between spectral performance with the number of antennas used at base station given in Figure 3 graph.

Figure 4 shows that the number of antennas grows spectral efficiency also increases in imperfect condition for MRC and ZF precoding technique and shows that SE increases as the number of antennas grow. All bounds are very tight. MRC and ZF both give a good performance. MRC works well for low SNIR and ZF for high SNIR.

Energy Efficiency performance for ZF and MRC precoding techniques with the number of antennas in uplink communication of massive MIMO system gives that user power requirement reduces as the number of antennas grows. In perfect CSI condition, it reduces by $1 / \mathrm{M}$ shown in the graph in Figure 5.

Figure 6 shows the imperfect CSI condition scale down by factor $1 / \sqrt{ } \mathrm{M}$.

Figure 7 showing the normalized power requirement to achieve spectral efficiency as $3 \mathrm{bit} / \mathrm{s} / \mathrm{Hz}$ per user as a 


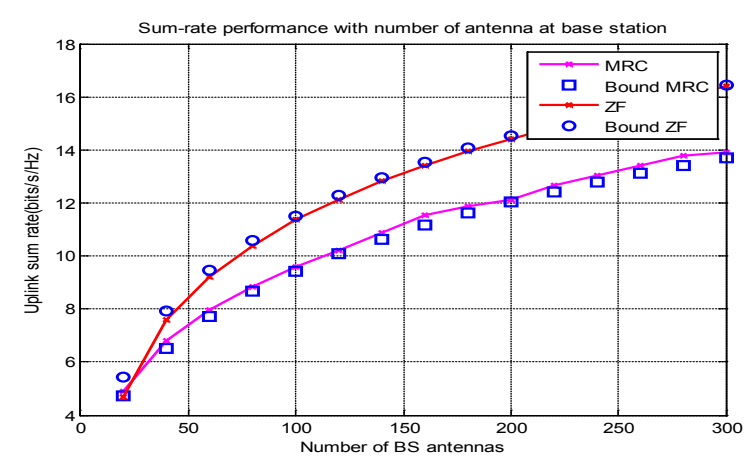

Figure 4. Spectral efficiency graph imperfect CSI with zero-forcing and maximal ratio combiner precoding technique.

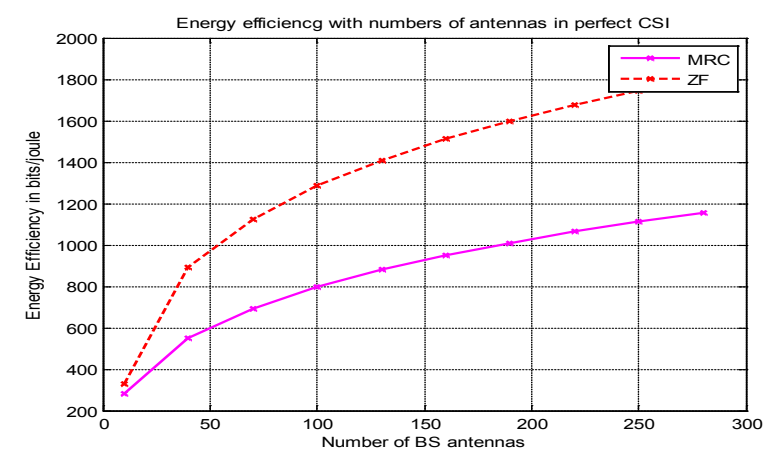

Figure 5. Energy efficiency graph for perfect CSI with zeroforcing and maximal ratio combiner precoding technique.

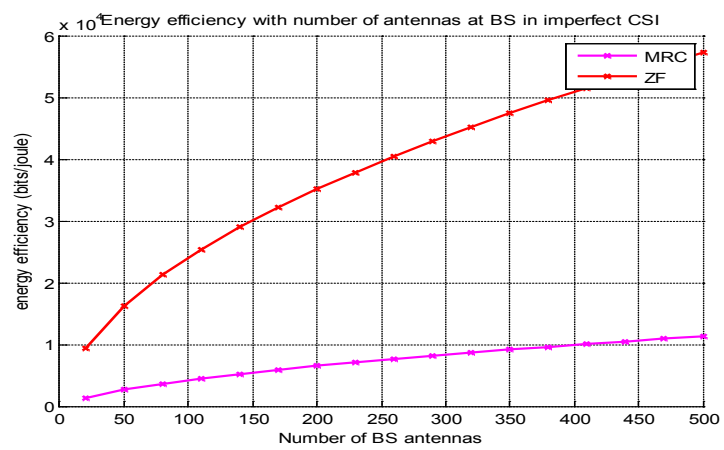

Figure 6. Energy efficiency graph for imperfect CSI with zero-forcing and maximal ratio combiner precoding technique.

function of $\mathrm{P}_{\mathrm{u}}$ with the number of antennas $(\mathrm{M})$. we can decrease $3 \mathrm{~dB}$ user power in case of perfect CSI by doubling the numbers of the antenna.

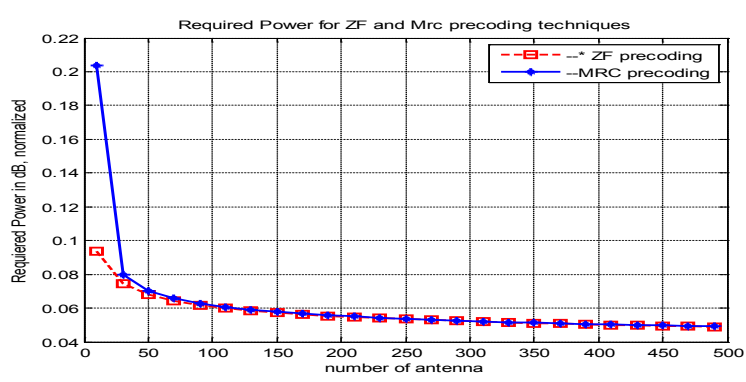

Figure 7. Required user power for spectral efficiency as 3 bits/s/Hz in perfect CSI condition with $\mathrm{T}=190$ and maximal ratio combiner precoding technique and $\tau=\mathrm{K}=10$ for $\mathrm{ZF}$ and MRC precoding in normalized $\mathrm{dB}$ form.

\section{Optimization for Energy Efficiency}

Multi-user large MIMO system when used in single-cell and multi-cell we can optimize parameter like user power $\left(P_{u}\right)$, number of uplink pilot carrier $(\tau)$, number of users $(\mathrm{K})$ and number of antenna $(\mathrm{M})$ for any required spectral efficiency. $\underline{\underline{Z}}$ When transmitting power $\left(\mathrm{P}_{\mathrm{u}}<<1\right.$ is very less so energy efficiency will depend on $\mathrm{M}, \mathrm{K}$ and $\tau . \underline{15}, \underline{16}$

$$
\begin{gathered}
\begin{array}{c}
A r g \max _{\tau, M, K} \eta=\sqrt{ }(((T \tau) / T) K \log 2(e) \\
\left.\tau(M-1) R_{i m p}^{\text {mrc }}\right)(M R C \text { Precoding })
\end{array} \\
\text { s.t. } R_{\operatorname{Imp}}^{M r c}=(((T-\tau) / T) K \log 2(e) \tau(M-1)) P_{u}{ }^{2} \\
\operatorname{Arg} \max _{\tau, M, K} \eta=\sqrt{ }(((T-\tau) / T) K \log 2(e) \\
\left.\tau(M-K) R_{\text {imp }}{ }^{z f}\right)(Z F \text { Precoding }) \\
\text { s.t. } R_{\operatorname{Imp}}^{Z F}=\left(((T-\tau) / T) K \log 2(e) \tau(M-K) P_{u}{ }^{2}\right.
\end{gathered}
$$

Optimizing $\mathbb{Q}$ in constrained spectral efficiency with small $\mathrm{P}_{\mathrm{u}}$. we can find maximum energy in constrain of constant spectral efficiency optimization of energy efficiency assumed that constant spectral efficiency is $3 \mathrm{bits} / \mathrm{Hz} / \mathrm{s}$ will give power requirement less than 1 and set chosen for optimization is Figures 8 and 9.

$$
1 \ll M \ll 500, K \leq \tau \leq T, 1 \leq K \ll M,
$$

the number of antennas and the number of users from the given set neglecting the large-scale fading (taken [D] $\left.{ }_{\mathrm{KK}}=\mathrm{I}_{\mathrm{K}}\right), \underline{2}, \underline{15}, \underline{10}, \underline{14}$ The energy efficiency is observed by a 3D graph for constant spectral efficiency. 


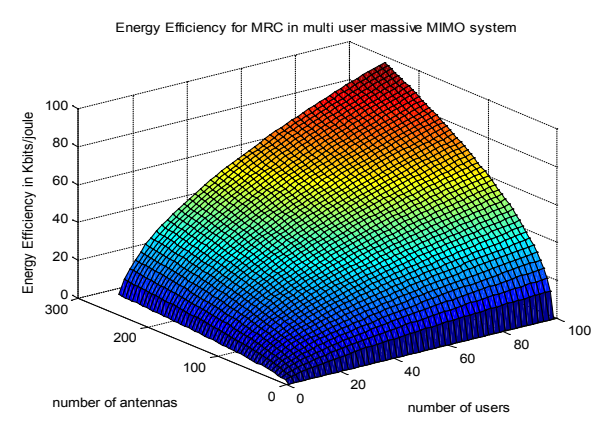

Figure 8. Optimized result of energy efficiency of MRC precoding for $3 \mathrm{bits} / \mathrm{Hz} / \mathrm{s}$ spectral efficiency.

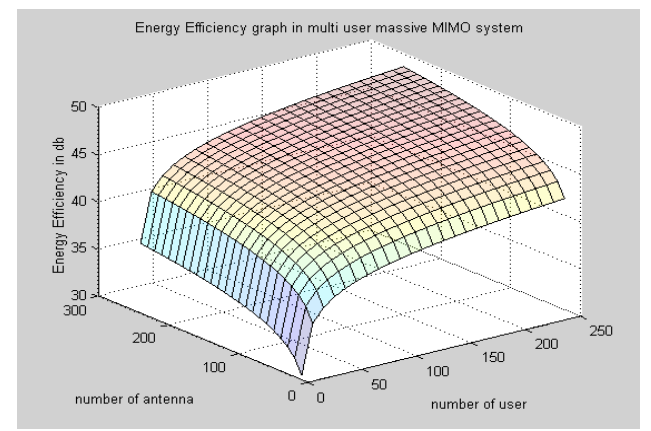

Figure 9. Optimized result of energy efficiency of ZF precoding for $3 \mathrm{bits} / \mathrm{Hz} / \mathrm{s}$ spectral efficiency.

\section{Conclusion}

We presented optimization for massive MIMO to select the number of antennas, the number of users and a minimum number of the pilot carrier to find the maximum value of energy efficiency. The study says spectral and energy efficiency can be bounded according to the requirement of the communication system. The huge antenna base station provides beamforming coverage. Our research result shows that $\mathrm{MRC}$ performs better in low power regime.

\section{References}

1. Han S, Xu Z, Rowell C. Large-scale antenna systems with hybrid analog and digital beamforming for millimeter wave 5G. IEEE Commun Mag. 2015;53(1):186-94.

2. Liu Z, Du W, Sun D. Energy and spectral efficiency tradeoff for massive MIMO systems with transmit antenna selection. IEEE Trans Veh Technol. 2017;66(5):4453-57.
3. Marzetta TL. Non cooperative cellular wireless with unlimited numbers of base station antennas. IEEE Trans Wirel Commun. 2010;9(11):3590-600.

4. EVD-based channel estimations for multi cell multiuser MIMO with very large antenna arrays. [cited 2012 Mar 25]. https://ieeexplore.ieee.org/document/6288608.

5. Ngo HQ, Larsson EG, Marzetta TL. Energy and spectral efficiency of very large multiuser MIMO systems. IEEE Trans Commun. 2013;61(4):1436-49.

6. Hong X, Jie Y, Wang C, Shi J, Ge X. Energy-spectral efficiency trade-off in virtual MIMO cellular systems. IEEE J Sel Areas in Commun. 2013:31(10):2128-40.

7. Xu J, Qiu L. Energy efficiency optimization for MIMO broadcast channels. IEEE Trans Wirel Commun. 2013;12(2):690-701.

8. Björnson E, Larsson EG, Debbah M. Massive MIMO for maximal spectral efficiency: how many users and pilots should be allocated? IEEE Trans Wirel Commun. 2016;15(2):1293-308.

9. Hoydis J. Massive MIMO in the UL/DL of cellular networks: how many antennas do we need? IEEE J Sel Areas Commun. 2013;31(2):160-71.

10. Xu W, Li S, Wang S, Feng Z, Lin J, Vasilakos A. Joint parameter selection for massive MIMO: an energy-efficient perspective. IEEE Access. 2016;4:3719-31.

11. Bjornson E, Sanguinetti L, Debbah M. Optimal design of energy-efficient multi-user MIMO systems: is Massive MIMO the answer? IEEE Trans Wirel Commun. 2015;14(6):3059-75.

12. Verenzuela D, Bjornson E, Sanguinetti L. Spectral and energy efficiency of superimposed pilots in uplink massive MIMO. IEEE Trans Wirel Commun. 2018;17(11):7099-115.

13. Wang Y, Li C, Huang Y, Wang D, Ban T, Yang L. Energyefficient optimization for DL massive MIMO FDD systems with transmit-side channel correlation. IEEE Trans Veh Technol. 2016;65(9):7228-43.

14. Heliot F, Imran MA, Tafazolli R. On the energy efficiency spectral efficiency trade-off over the MIMO Rayleigh fading channel. IEEE Trans Commun. 2012;60(5):1345-56.

15. Ku I, Wang C, Thompson JS. Spectral-energy efficiency tradeoff in relay-aided cellular networks, IEEE Trans Wirel Commun. 2013;12(10):4970-82.

16. Vieira J, Rusek F, Edfors O, Malkowsky S, Liu L, Tufvesson F. Reciprocity calibration for massive mimo: proposal, modeling, and validation. IEEE Trans Wirel Commun. 2017;16(5):3042-56. 